\title{
Critères de détection indirecte des taureaux porteurs de la translocation 1-29 à partir des caryotypes de leurs descendants
}

\author{
J.L. FOULLEY et J. FREBLING \\ I.N.R.A., Station de Génétique quantitative et appliquée \\ Centre de Recherches Zootechniques, F 78350 Jouy-en-Josas
}

\begin{abstract}
Résumé
Cet article donne, à partir du théorème de Bayes, l'expression de la probabilité qu'un taureau non caryotypé soit porteur de la translocation 1-29 sachant qu'on a observé $\mathrm{S}$ descendants porteurs parmi $\mathbf{N}$ caryotypés. Les formules sont illustrées par un exemple concret et des tables indiquant le nombre minimum de porteurs requis pour conclure avec un risque de décision erronée inférieure à $10^{-3}$ ou $10^{-6}$.
\end{abstract}

Mots clés : Bovins, translocation 1-29, théorème de Bayes.

\author{
Summary \\ Criteria for the indirect detection of the 1-29 translocation \\ in bulls using their karyotyped progeny
}

Using Bayes theorem, this paper presents the expression of the probability that a non-karyotyped bull can carry the 1-29 translocation when Y carriers have been observed among N-karyotyped progeny. The formulas have been illustrated by concrete examples, and the tables show the minimum number of carriers needed to reach correct decision with a risk of less than $10^{-3}$ or $10^{-6}$.

Key words : Cattle, 1-29 translocation, Bayes'theorem.

\section{Introduction}

La translocation 1-29 est l'anomalie chromosomique la plus fréquente chez les bovins (POPESCU, 1977 ; CrIBIU, 1980). Hormis certaines races à diffusion importante qui en sont indemnes (souches Pie-Noir dont Holstein, Normande, Hereford), la plupart des populations exploitées dans le monde pour le lait ou pour la viande sont touchées par cette anomalie. Celle-ci, de par la formation de gamètes déséquilibrés à la méiose induit une baisse très sensible de fertilité, notamment chez les 
femelles (Gustavsson, 1969 ; ReFsDal, 1976). Aussi certains pays ont proposé des programmes de caryotypage et d'éradication notamment des mâles destinés à une utilisation en insémination artificielle.

En fait, tous les taurillons impliqués dans les programmes de sélection des centres d'insémination ne sont pas caryotypés, soit qu'il n'existe pas de programme de dépistage systématique, soit que celui-ci soit trop récent pour toucher l'ensemble des taureaux dont la semence est mise à la disposition des éleveurs. De plus, il ne faut jamais exclure des causes accidentelles (mort du taureau, prélèvement de sang défectueux) de manque d'information. Pour toutes ces raisons, il est utile de prévoir des critères de détection indirecte des porteurs, ainsi qu'il est de règle pour certaines anomalies chez les bovins (S.A.P. ; Lauvergne \& Faucon, 1976). Cette note présente des critères numériques simples de décision basés sur les résultats caryotypiques de descendants.

\section{Méthode}

Le problème peut se formuler ainsi : on dispose de l'information caryotypique sur un échantillon aléatoire de $\mathrm{N}$ descendants (demi-frères, sœurs entre eux) d'un taureau de caryotype inconnu. A partir de combien de descendants porteurs peut-on raisonnablement en inférer que le père était porteur. Il s'agit d'un problème similaire à ceux rencontrés dans les études de présomption de paternité à partir des données de groupes sanguins (HURON \& RUfFí, 1959). Comme dans ceux-ci, on aura recours au théorème de Bayes (LindeY, 1965) écrit, en l'occurrence sous la forme suivante :

où :

$$
\mathrm{P}\left(\mathrm{C}_{i} / \mathrm{A}\right)=\frac{\mathrm{P}\left(\mathrm{C}_{i}\right) \mathrm{P}\left(\mathrm{A} / \mathrm{C}_{i}\right)}{\sum_{i} \mathrm{P}\left(\mathrm{C}_{i}\right) \mathrm{P}\left(\mathrm{A} / \mathrm{C}_{i}\right)}
$$

- $\mathrm{C}_{i}$ désigne les différents états caryotypiques possibles du père soit ici homozygote porteur (TT), hétérozygote porteur $(\mathrm{Tt})$ et homozygote normal $(\mathrm{tt})$.

- A les observations caryotypiques faites sur les $\mathbf{N}$ descendants.

- $\mathrm{P}\left(\mathrm{C}_{i}\right)$ les probabilités a priori des états caryotypiques dans la population que nous noterons $\mathrm{C}_{i}$ avec $i=\mathrm{TT}$, Tt ou $\mathrm{tt}$.

- $\mathbf{P}\left(\mathrm{A} / \mathrm{C}_{i}\right)$ est la probabilité conditionnelle de réalisation des observations $\mathrm{A}$ sachant que le père était dans l'état $C_{i}$.

De façon générale, si les caryotypes des $\mathrm{N}$ descendants d'un mème père peuvent être considérés comme indépendants, ce qui est le cas s'il n'y a pas d'accouplements préférentiels entre pères et mères vis-à-vis de la translocation ou d'un caractère lié et si l'on observe un échantillon non sélectionné de $\mathbf{N}$ descendants génétiquement indépendants tels que par exemple des demi-germains paternels issus de mères différentes choisies au hasard, la loi de distribution conditionnelle de ces $\mathrm{N}$ informations caryotypiques en des nombres (X, Y et $\mathrm{Z}$ ) des 3 catégories possibles (TT, Tt et $t$ ) est une loi multinominale telle que :

$$
\mathbf{P}\left(\mathrm{A} / \mathrm{C}_{i}\right)=\frac{\mathrm{N} !}{\mathrm{X} ! \mathrm{Y} ! \mathrm{Z} !}\left[\mathrm{P}\left(\mathrm{TT} / \mathrm{C}_{i}\right)\right]^{\mathrm{X}}\left[\mathrm{P}\left(\mathrm{Tt} / \mathrm{C}_{i}\right)\right]^{\mathrm{Y}}\left[\mathrm{P}\left(\mathrm{tt} / \mathrm{C}_{i}\right)\right]^{\mathrm{Z}}
$$


TABlEAU 1

Tableau de probabilités des caryotypes d'un descendant en fonction de celui du père. Probabilities of the different karyotypes of the progeny when those of the sire are known.

\begin{tabular}{|c|c|c|c|c|}
\hline \multirow{2}{*}{$\begin{array}{l}\text { Caryotype possible } \\
\text { du père }\end{array}$} & \multirow{2}{*}{$\begin{array}{c}\text { Probabilité } \\
\text { a priori }\end{array}$} & \multicolumn{3}{|c|}{ Probabilité qu'un descendant soit } \\
\hline & & TT & $\mathrm{Tt}$ & $\mathrm{tt}$ \\
\hline Homozygote porteur (TT) & $\Pi_{\mathrm{TT}}$ & $\mathrm{q}_{\mathrm{f}}$ & $1-\mathrm{q}_{\mathbf{f}}$ & 0 \\
\hline Hétérozygote porteur $(\mathrm{Tt})$ & $\Pi_{T t}$ & $1 / 2 q_{f}$ & $1 / 2$ & $1 / 2\left(1-q_{p}\right)$ \\
\hline Homozygote normal $(\mathrm{tt})$ & $\Pi_{\mathrm{tt}}$ & 0 & $\mathrm{q}_{\mathrm{f}}$ & $1-q_{f}$ \\
\hline
\end{tabular}

$\Pi$ : Fréquence d'un état caryotypique chez le père avec $\Pi_{\mathrm{TT}}+\Pi_{\mathrm{Tt}}+\Pi_{\mathrm{tt}}=1$.

q : Fréquence des chromosomes 1-29 «attachés» par rapport aux chromosomes 1 libres.

Les probabilités des 3 caryotypes de descendants conditionnellement à celui $\left(C_{i}\right)$ du père sont données au tableau 1 sous forme d'une matrice de transition de LI (LI \& SACKS, 1954) en fonction de la fréquence génique $\left(\mathrm{q}_{\mathrm{f}}\right)$ de l'anomalie dans la population femelle commune. La translocation 1-29 peut, en effet être considérée comme un caractère mendelien simple autosomal. Dans ce cadre, on peut interpréter la fréquence génique de l'anomalie comme la fraction de chromosomes 1-29 fusionnés par rapport aux chromosomes 1 libres. Du tableau 1 et du théorème de Bayes (formule 1), on déduit immédiatement les expressions générales des probabilités que le père soit de génotype $\mathrm{TT}, \mathrm{Tt}$ et $\mathrm{tt}$ respectivement sachant qu'on a observé $\mathrm{X}, \mathrm{Y}, \mathrm{Z}$ descendants (demi-germains paternels entre eux) de type TT, Tt et tt respectivement.

avec :

$$
\begin{aligned}
& \mathrm{P}(\mathrm{TT} / \mathrm{X}, \mathrm{Y}, \mathrm{Z})=\left[\Pi_{\mathrm{TT}} \cdot \mathrm{q}_{\mathrm{f}} \mathrm{X} \cdot\left(1-\mathrm{q}_{\mathrm{f}}\right)^{\mathrm{Y}} \cdot \mathrm{O}^{\mathrm{Z}}\right] / \mathrm{D} \\
& \mathrm{P}(\mathrm{Tt} / \mathrm{X}, \mathrm{Y}, \mathrm{Z})=\left[\Pi_{\mathrm{Tt}} \cdot(1 / 2)^{\mathrm{N}} \cdot \mathrm{q}_{\mathrm{f}} \mathrm{X} \cdot\left(1-\mathrm{q}_{\mathrm{f}} \mathrm{Z}\right] / \mathrm{D}\right. \\
& \mathrm{P}(\mathrm{tt} / \mathrm{X}, \mathrm{Y}, \mathrm{Z})=\left[\Pi_{\mathrm{tt}} \cdot 0^{\mathrm{X}} \cdot \mathrm{q}_{\mathrm{f}}^{\mathrm{Y}}\left(1-\mathrm{q}_{\mathrm{f}}\right)^{\mathrm{Z}}\right] / \mathrm{D}
\end{aligned}
$$

- D égal à la somme des numérateurs des expressions (3) ;

- $\mathrm{N}=\mathrm{X}+\mathrm{Y}+\mathrm{Z}$;

- $0^{\alpha}=0$ si $\alpha>0$ et 1 si $\alpha=0$.

Ces formules s'appliquent de façon générale à toutes les situations qui peuvent se rencontrer dans le cadre d'une information caryotypique sur $\mathbf{N}$ demi-germains paternels.

En pratique toutefois, il peut être utile d'envisager différentes situations particulières qui découlent de 2 cas évidents d'exclusion au vu du tableau 1 (ou des formules 3), à savoir :

- s'il y a un descendant non porteur $(Z \neq 0)$, le père ne peut être homozygote porteur : $\mathbf{P}(\mathrm{TT} / \mathrm{X}, \mathrm{Y}, \mathrm{Z} \neq 0)=0$;

- l'observation d'un descendant porteur homozygote $(X \neq 0)$ exclut que le père puisse être normal : $\mathrm{P}(\mathrm{tt} / \mathrm{X} \neq 0, \mathrm{Y}, \mathrm{Z})=0$.

Cette dernière condition constitue une dichotomie évidente dans les règles de décision. L'application du théorème de Bayes va donc concerner essentiellement les situations d'incertitude où ne se rencontre pas de porteur homozygote 1-29 parmi les descendants caryotypés $(X=0)$. 
Dans ce cas, la probabilité que le père soit porteur de l'anomalie (à l'état homozygote ou hétérozygote) s'obtient simplement par l'addition des formules ( $3 \mathrm{a}$ ) et ( $3 \mathrm{~b}$ ) avec la valeur de $\mathrm{X}$ mise à zéro.

$$
\mathrm{P}(\mathrm{TT} \text { ou } \mathrm{Tt} / \mathrm{X}=0, \mathrm{Y}, \mathrm{N}-\mathrm{Y})=(3 \mathrm{a})+(3 \mathrm{~b}) \text { avec } \mathrm{X}=0
$$

En pratique va se présenter fréquemment le cas où il y aura au moins un caryotype normal parmi les descendants $\left(\mathrm{Z}=\mathrm{N}-\mathrm{Y} \neq 0\right.$; $\mathrm{cf}$. $1^{\text {er }}$ cas d'exclusion) qui restreint le calcul à celui de la probabilité que le père soit porteur hétérozygote de la translocation. La formule se simplifie et s'écrit alors :

$$
\mathbf{P}(\mathrm{Tt} / 0, \mathrm{Y}, \mathrm{N}-\mathrm{Y} \neq 0)=\frac{\Pi_{\mathrm{Tt}}}{\Pi_{\mathrm{Tt}}+\left(\Pi_{\mathrm{tt}} \cdot \mathrm{q}_{\mathrm{f}} \mathrm{Y} \cdot 2^{\mathrm{N}}\right)}
$$

Si l'information fournie sur les descendants ne précisait pas l'état homozygote ou hétérozygote des porteurs, la formule précédente ne serait plus valable et il faudrait appliquer la variante suivante :

$$
P(T t / Y, N-Y \neq 0)=\frac{\Pi_{T t} \cdot\left(1+q_{f}\right)^{Y}}{\left[\Pi_{T t} \cdot\left(1+q_{f}\right)^{Y}\right]+\left(\Pi_{t t} \cdot q_{f}{ }^{Y} \cdot 2^{N}\right)}
$$

Si l'on envisage maintenant, la probabilité que le père puisse être porteur homozygote, on s'intéressera à cette probabilité, soit a priori $(\mathrm{Z}=0)$, soit le plus souvent, conditionnellement au fait que le père soit porteur. Un père ne peut être a priori reconnu porteur sans ambiguité que si tous ses descendants le sont $(Z=0)$ et l'un d'entre eux au moins est homozygote $(X \neq 0)$.

Cette probabilité s'exprime alors, quel que soit $X \geqslant 1$, par :

$$
\mathbf{P}(\mathrm{TT} / \text { père porteur } \mathrm{X}, \mathrm{N}-\mathrm{X}, 0)=\frac{\Pi_{\mathrm{TT}} \cdot\left(1-\mathrm{q}_{\mathrm{f}}\right)^{\mathrm{N}-\mathrm{X}}}{\left[\Pi_{\mathrm{TT}} \cdot\left(1-\mathrm{q}_{\mathrm{f}}\right)^{N-X}\right]+\left(\Pi_{\mathrm{Tt}} / 2^{N}\right)}
$$

L'ensemble des situations particulières étudiées et la logique de leur présentation basée sur les 2 cas d'exclusion $(X \neq 0 \mathrm{P}(\mathrm{tt})=0 ; \mathrm{Z} \neq 0 \mathrm{P}(\mathrm{TT})=0)$ sont résumées au tableau 2.

\begin{tabular}{|c|c|c|c|}
\hline & & \multicolumn{2}{|c|}{ Nombre $\mathrm{Z}$ de descendants homozygotes normaux } \\
\hline & & $\begin{array}{c}\mathrm{Z} \neq 0 \\
\text { Exclusion d'un père } \mathrm{TT}\end{array}$ & $\mathrm{Z}=0$ \\
\hline \multirow{2}{*}{$\begin{array}{c}\text { Nombre X } \\
\text { de descendants } \\
\text { homozygotes } \\
1-29\end{array}$} & $\begin{array}{c}\mathrm{X} \neq 0 \\
\text { Exclusion } \\
\text { d'un père } \mathrm{tt}\end{array}$ & $\begin{array}{l}P(T T)=0 \\
P(T t)=1 \\
P(t)=0\end{array}$ & $\begin{array}{l}\mathrm{P}(\mathrm{TT}) \neq 0: \text { formule }(7) \\
\mathrm{P}(\mathrm{Tt}) \neq 0 \\
\mathrm{P}(\mathrm{tt})=0\end{array}$ \\
\hline & $\mathrm{X}=0$ & $\begin{array}{l}\mathrm{P}(\mathrm{TT})=0 \\
\mathrm{P}(\mathrm{Tt}) \neq 0: \text { formule }(5) \\
\mathrm{P}(\mathrm{tt}) \neq 0\end{array}$ & $\begin{array}{l}\mathrm{P}(\mathrm{TT}) \neq 0 \\
\mathrm{P}(\mathrm{Tt}) \neq 0 \\
\mathrm{P}(\mathrm{tt}) \neq 0\end{array}$ \\
\hline
\end{tabular}

\section{TABLEAU 2}

Situations particulières étudiées.

Particular situations studied. 


\section{Résultats}

A. Les formules précédentes permettent de calculer cas par cas la probabilité qu'au taureau donné soit porteur de l'anomalie 1-29 à l'état hétérozygote ou homozygote, compte tenu de l'information obtenue sur ses descendants, notamment de testage.

A titre d'illustration, on peut rapporter l'exemple d'un taureau de race à viande utilisé largement en insémination artificielle dont le caryotype n'avait pu être réalisé, mais dont 29 fils et filles, demi-germains entre eux ont pu être typés parmi lesquels 13 se sont avérés porteurs hétérozygotes (DARRE, 1984, communication personnelle). Ce cas est redevable de l'application de la formule (5). La fréquence de l'anomalie chez les mâles d'IA est relativement bien connue dans cette race. Une valeur de 0,125 a été retenue pour la fréquence génique $q_{m}$ d'où on a déduit les fréquences de porteurs hétérozygotes $\left(\Pi_{\mathrm{Tt}}\right)$ et de normaux $\left(\Pi_{\mathrm{tt}}\right)$ par les formules de HardyWeinberg en supposant que $q_{m}$ est la fréquence génique chez les pères et chez les mères à taureaux soit :

- $\Pi_{\mathrm{Tt}}=2 \times 0,125 \times 0,875=0,21875$

- $\Pi_{\mathrm{tt}}=(0,875)^{2}=0,765625$

Bien qu'on ait actuellement peu d'éléments pour l'apprécier, la fréquence génique de l'anomalie chez les femelles communes est probablement plus faible que chez les mâles. $\mathrm{Si}$ on prend une valeur de $\mathrm{q}_{\mathrm{f}}$ égale à 0,075 , on trouve alors par application de la formule (5) :

$$
P=\frac{0,21875}{0,21875+\left(0,765625 \times 0,075^{13} \times 2^{299}\right)}=0,9999955 .
$$

Même dans l'hypothèse très improbable où la fréquence de l'anomalie serait aussi élevée chez les femelles que chez les mâles (cas de femelles mères à taureaux), la probabilité que le père soit porteur hétérozygote reste élevée $(P=0,9966)$; on peut donc conclure avec un risque minime de diffusion d'une information erronée préjudiciable au centre d'insémination artificielle que le taureau en question bien que non caryotypé est porteur hétérozygote de l'anomalie.

B. Une autre voie d'utilisation des formules est l'établissement de tables à des fins, par exemple, de diffusion réglementaire de l'information sur l'anomalie. Dans ce cas, il faut se fixer d'une part les valeurs des fréquences géniques $q_{m}$ et $q_{f}$ des mâles et des femelles à utiliser dans la race concernée et, d'autre part, le seuil de probabilité Po à partir duquel le père sera déclaré porteur. Des exemples de telles tables ont été ébauchés et proposés à la réflexion aux tableaux 3 et 4 en vue de la détection de pères porteurs hétérozygotes et homozygotes respectivement.

Les seuils donnés au tableau 3 du nombre de porteurs hétérozygotes parmi $\mathrm{N}$ descendants caryotypés à partir desquels le père sera déclaré porteur (TT ou $\mathrm{Tt}$ ) ont été établis pour des fréquences de 1,$25 ; 2,5 ; 3,75 ; 5 ; 7,5 ; 10$ et 12,5 p. 100 . Ces chiffres correspondent à la gamme des situations rencontrées dans les races mixtes et à viande françaises vis-à-vis de la translocation avec les races peu touchées $(\mathrm{q} \leqslant 2,5$ p. 100$)$ telles la Montbéliarde (2,5 p. 100 environ de porteurs) et la Charo- 


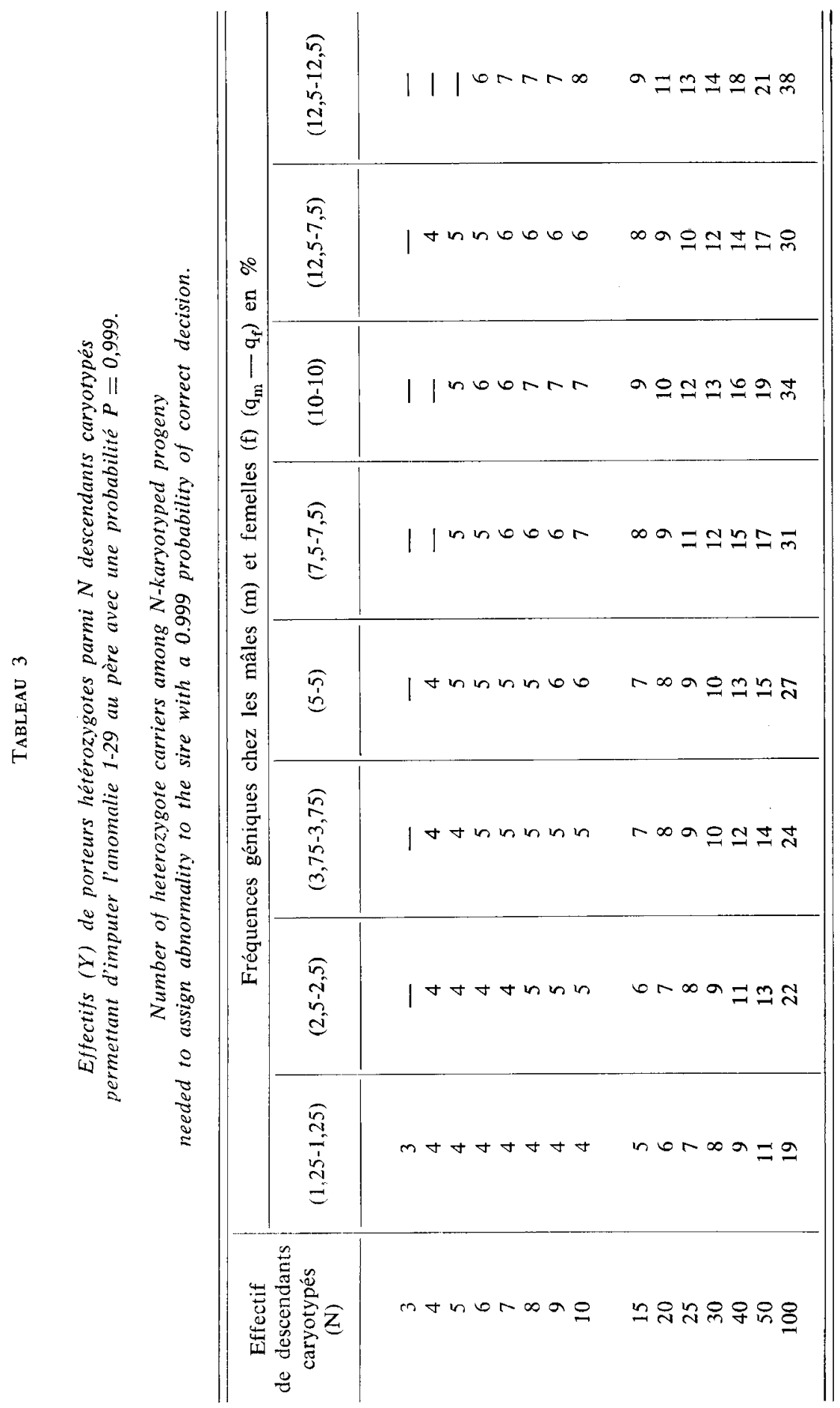


laise ( 3 ou 4 p. 100 de porteurs environ) puis celles moyennement touchées $(3,75$ p. 100 $\leqslant \mathrm{q} \leqslant 5$ p. 100$)$ telles la Limousine (7,5 p. 100 environ de porteurs), la Gasconne $(8,5$ p. 100 environ de porteurs) et une souche spécialisée de croisement $(10$ p. 100 de porteurs) et au-delà $(7,5 \leqslant q \leqslant 12,5$ p. 100$)$, les races sévèrement touchées. La fraction minimum de porteurs par rapport aux caryotypés qui doit être observée est d'autant plus élevée que l'effectif $\mathbf{N}$ de caryotypés est faible. Il y a même un plancher pour $\mathbf{N}$ de 4 à 6 dans la gamme de fréquences étudiées et pour un risque maximum de 1/1000 en deçà duquel il serait hasardeux de conclure.

A effectif $\mathrm{N}$ égal de caryotypés, le seuil $\mathrm{Y}$ est d'autant plus élevé que la fréquence génique de l'anomalie augmente. Avec 10 descendants caryotypés par exemple et un risque de décision erronée de 1 pour 1000 il suffit d'observer 6 porteurs à $q=5$ p. 100 alors qu'il en faut au moins 8 à $q=25$ p. 100 .

Il faut distinguer l'incidence de la variation de la fréquence chez les mâles d'IA de celle chez les femelles notamment celles support du testage. A fréquence génique constante des mâles, les effectifs requis de porteurs augmentent logiquement comme le laisse prévoir le bon sens ( 1 porteur suffit quand $\mathrm{q}_{f}=0$ ) avec la fréquence de l'anomalie chez les femelles. Les dernières colonnes du tableau 3 le montrent clairement ; pour $\mathrm{N}=25$ on passe de $\mathrm{Y}=10$ à 13 porteurs quand la fréquence génique chez les femelles passe de 7,5 à 12,5 p. 100 , la fréquence chez les mâles étant inchangée à 12,5 p. 100 . La fréquence génique chez les mâles intervient uniquement à travers les poids a priori des 2 éventualités "père porteur hétérozygote » et "père non porteur» qui sont envisagées dans le théorème de Bayes. On suppose pour simplifier que la fréquence génique est identique et égale à $\mathrm{q}_{\mathrm{m}}$ chez les pères et les mères à taureaux supposés accouplés au hasard, le calcul des probabilitées a priori $\Pi$ découlant alors de la formule de Hardy-Weinberg. Toutes choses égales par ailleurs, notamment la fréquence génique de l'anomalie chez les femelles, la probabilité que le père soit porteur hétérozygote augmente avec la fréquence chez les mâles. A un seuil donné de probabilité et pour un effectif fixé $\mathbf{N}$ de descendants caryotypés, le nombre de porteurs requis diminue donc avec la fréquence de l'anomalie chez les mâles contrairement à ce qui se passait lors d'une variation de cette fréquence chez les femelles. La variation observée ici pour un seuil de $P=0,999$ est très faible comme le montre très bien la comparaison du tableau 3 des colonnes relatives à $\left(q_{m}, q_{f}\right)=$ $(0,075 ; 0,075)$ et $(0,125 ; 0,075)$. Au-delà de $N=15$ pour les races peu et moyennement touchées $(\mathrm{q} \leqslant 5$ p. 100$)$ et $\mathrm{N} \simeq 25$ pour celles sévèrement touchées $(\mathrm{q}$ de 7,5 à 12,5 p. 100), le seuil de porteurs requis descend nettement en dessous de 50 p. 100. Ce chiffre pourrait, à première vue, étonner puisqu'au fond on observe l'apparition d'un gène dominant dans la descendance d'un individu hétérozygote.

Quoiqu'il en soit, les effectifs de descendants à caryotyper ne sont pas irréalistes à envisager pour des taureaux d'insémination artificielle impliqués dans des programmes de contrôle sur descendance où, même en station (cas le plus défavorable) 20 à 25 produits sont contrôlés. Les effectifs minima de descendants porteurs à observer rendent la détection indirecte des pères homozygotes, reconnus a priori porteurs relativement difficiles (tabl. 4). Pour $X=1$, l'effectif minimum de porteurs requis ne dépend pratiquement pas de la fréquence génique dans la zone des fréquences étudiées : $N=17$ pour $P=0,999$. Enfin, si l'on veut vraiment réduire à un niveau infinitésimal le risque d'imputation à tort de l'anomalie à l'état homozygote, les effectifs minima requis de caryotypés porteurs augmentent de façon appréciable : $\mathrm{N}=28$ à 30 pour $\mathrm{P}=1-10^{6}$ et $\mathrm{X}=1$. 


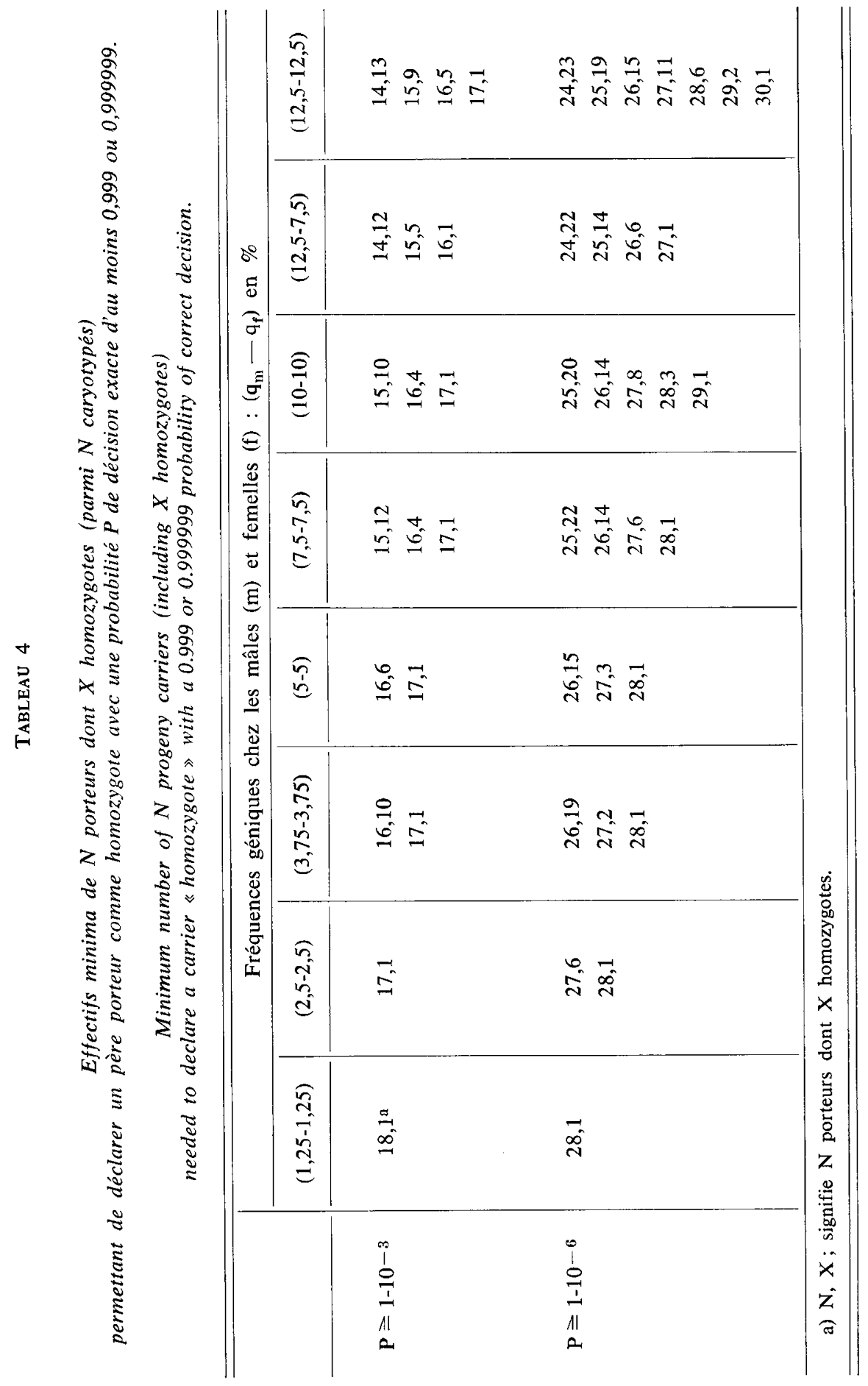




\section{Discussion - Conclusion}

A. Les calculs présentés supposent que l'anomalie est uniquement héritée et qu'il n'y a pas d'apport dû à des mutations récurrentes. Dans l'éventualité contraire, on pourra s'inspirer utilement de l'approche développée par MurPhy \& MutaliK (1969). Par ailleurs, on a admis que la distribution des porteurs dans l'échantillon caryotypé ne dépend que des probabilités de transmission des "gènes » et n'est donc pas influencée par des effets sélectifs indirects dus aux choix zootechniques qui peuvent précéder le caryotypage. Les hypothèses simples adoptées ici peuvent être bien entendu sujettes à discussion (Chevalet et al., 1984). Toutefois, en l'absence de faits expérimentaux manifestes, elles ne paraissent pas devoir être remises en cause dans le cadre de ce travail.

B. On n'a considéré que la situation simple d'informations caryotypiques provenant de descendants demi-germains paternels de première génération. D'autres cas plus complexes pourraient être envisagés en appliquant la même méthodologie. Avec les schémas actuels de sélection des bovins, on pourra par exemple rencontrer le cas de mâles non caryotypés mais pour lesquels on possède une information sur les demi-frères paternels au stade du contrôle individuel. Il suffira alors de calculer avec les formules (3) la probabilité que leur père soit des types TT, Tt et tt respectivement pour obtenir ensuite celle d'un type donné du fils de caryotype inconnu en question grâce aux coefficients du tableau 1. De façon générale, si $\mathbf{P}$ est le vecteur de probabilité des 3 types $\mathrm{TT}$, Tt et tt respectivement, la relation à utiliser s'écrit :

$$
\mathbf{P} \text { individu }=\mathbf{M}^{\prime} \cdot \mathbf{P} \text { père }
$$

où $M^{\prime}$ est la matrice transposée de la matrice de transition $\mathbf{M}(3,3)$ donnée au tableau 1.

$\mathrm{Si}$, ultérieurement des descendants de cet individu sont caryotypés, cette information pourra être cumulée à la précédente sur les demi-frères ; il suffira de remplacer dans les formules (3), (5) et (7) les probabilités $\Pi$ a priori des 3 caryotypes de l'individu par celles homologues conditionnelles à la première information sur les demifrères.

C. Enfin, une des critiques qu'on pourrait adresser à ces formules réside dans la difficulté d'un choix réaliste des fréquences géniques. Même s'il n'existe pas de statistiques locales, la bibliographie fournit des renseignements sur les principales races utilisées dans le monde, notamment en ce qui concerne les mâles d'insémination artificielle. Pourvu que le seuil de probabilité de détection d'un père hétérozygote porteur soit suffisamment élevé, un chiffre approché, voire un ordre de grandeur sera largement suffisant à ce niveau de la fréquence génique de l'anomalie chez les mâles, comme cela a été montré précédemment. Par contre, des statistiques d'envergure sont très rares chez les femelles et l'incidence d'une variation de la fréquence génique est ici beaucoup plus marquée. Une borne supérieure vraisemblable est la valeur rencontrée chez les mâles avant mise en place d'une politique d'éradication. Comme le nombre de porteurs requis augmente avec la fréquence chez les femelles, une 
solution de sécurité (vis-à-vis du préjudice causé au centre d'IA par une décision erronée) est donc de prendre la même valeur que chez les mâles, tout au moins dans les races peu ou moyennement touchées $\left(q_{n} \leqslant 0,05\right)$.

Reçu pour publication le 10 août 1984. Accepté pour publication le 15 janvier 1985.

\section{Références bibliographiques}

Chevalet C., De Rochambeau H., Vu Tien Khang Jacqueline, 1984 . - Insémination artificielle et gestion de la variabilité génétique. Les colloques de l'I.N.R.A. : Séminaire des départements de génétique et physiologie animales de l'I.N.R.A., Toulouse-Auzeville, 23-24 novembre 1983, Insémination artificielle et Amélioration génétique : Bilan et perspectives critiques, I.N.R.A., Versailles.

Cribiu E.P., POPESCU C.P., 1980. Distribution of the 1/29 Robertson translocation in France. Proceedings of the iV European Colloquium on Cytogenetics of Domestic Animals, Uppsala, June 10-13, 1980, 130-135.

Gustavsson I., 1969. Cytogenetics, distribution and phenotypic effects of a translocation in Swedish cattle. Hereditas, 63, 68-169.

Huron R., Ruffié J., 1959. Les méthodes en génétique générale et génétique humaine. 556 pp., Masson, Paris.

Lauvergne J.J., Faucon A., 1976. Le syndrome d'Arthrogrypose et de Palatoschisis (S.A.P.) en race bovine Charolaise. Bibliographie annotée 1967-1975. Ann. Génét. Sél. Anim., 8, $51-70$

Li C.C., SACKS L., 1954. The derivation of joint distribution and correlation between relatives by the use of stochastic matrices. Biometrics, 10, 347-360.

Lindey D.V., 1965. Introduction to Probability and Statistics. Part 1. Probability. 1980 edition, 259 pp., Cambridge University Press, Cambridge.

MURPHY E.A., MUTAliK G.S., 1969. The application of Bayesian methods in genetic counselling. Hum. Hered., 19, 126-151.

Popescu C.P., 1977. Les anomalies chromosomiques des bovins (Bos taurus L.). Etat actuel des connaissances. Ann. Génét. Sél. Anim., 9, 463-470.

Refsdal A.O., 1976. Low fertility in daughters of bulls with 1-29 translocation. Acta Vet. Scand., 17, 190-195. 